\title{
Utilization of Chinese medicine for respiratory discomforts by patients with a medical history of tuberculosis in Taiwan
}

\author{
Su-Tso Yang ${ }^{1,2^{*}+}$, Yi-Rong Lin ${ }^{1}$, Mei-Yao Wu ${ }^{3}$, Jen-Huai Chiang ${ }^{4}$, Pei-Shan Yang ${ }^{3}$, Te-Chun Hsia ${ }^{5,6}$ and \\ Hung-Rong Yen ${ }^{1,3,7,8,9,10^{*}+}$ (D)
}

\begin{abstract}
Background: Tuberculosis (TB) is one of the world's major communicable infectious diseases, and it still imposes a great health burden in developing countries. The development of drug-resistant TB during the treatment increases the treatment complexity, and the long-term pulmonary complications after completing treatment raise the epidemic health burden. This study intended to investigate the utilization of Chinese medicine (CM) for respiratory symptoms by patients with a medical history of TB in Taiwan.

Methods: We analyzed a cohort of one million individuals who were randomly selected from the National Health Insurance Research Database in Taiwan. The inclusion criteria of patients $(n=7905)$ with history of TB (ICD-9-CM codes 010-018 and A02) were: (1) TB diagnosed between January 1, 1997 and December 31, 2010 (2) 18 years old or over (3) Clinical records for at least 2 months with complete demographic information (4) Record of treatment with first-line TB medication prescriptions. CM users for conditions other than respiratory discomforts $(n=3980)$ were excluded. Finally, a total of 3925 TB patients were categorized as: CM users for respiratory discomforts $(n=2051)$ and non-CM users ( $n=1874)$.

Results: Among the 3925 subjects, 2051 (52.25\%) were CM users, and 1874 (44.753\%) were non-CM users. Female patients and those who were younger (18-39 y/o) and who lived in urbanized areas relatively tended to be CM users $(p<.0001)$. Most of the CM users $(1944,94.78 \%)$ received Chinese medicines. The most commonly prescribed herbal formulas and single herbs were Xiao-Qing-Long-Tang and Radix Platycodonis (Jie-Geng), respectively. The core pattern of Chinese medicines for TB patients consisted of Ma-Xing-Gan-Shi-Tang, Bulbus Fritillariae Thunbergii (Bei-Mu), Radix Platycodonis (Jie-Geng) and Semen Armeniacae (Xing-Ren).
\end{abstract}

Conclusions: The use of CM is popular among patients with a medical history of TB complicated with long-term respiratory discomforts in Taiwan. Further pharmacological investigations and clinical trials are required.

Keywords: Chinese medicine, National Health Insurance Research Database, Prescription, Respiratory diseases, Tuberculosis

\section{Introduction}

In the twenty-first century, tuberculosis (TB) continues to be one of the world's major health challenges. TB is a deadly communicable disease that is primarily transmitted from human to human by droplet infection, and it is a major health burden in both developed and developing

\footnotetext{
* Correspondence: yangst@mail.cmu.edu.tw; hungrongyen@gmail.com; hungrongyen@mail.cmu.edu.tw

${ }^{+}$Su-Tso Yang and Hung-Rong Yen contributed equally to this work. ${ }^{1}$ Graduate Institute of Chinese Medicine, School of Chinese Medicine, College of Chinese Medicine, China Medical University, Taichung, Taiwan Full list of author information is available at the end of the article
}

countries. Approximately 9.0 million people developed $\mathrm{TB}$, and 1.5 million died from the disease in 2013 [1]. Of the 9.0 million estimated cases, $56 \%$ were in Southeast Asian and Western Pacific regions [2]. China accounted for $11 \%$ of the 9.0 million estimated TB cases, and 11,528 TB cases were confirmed in Taiwan [3]. Male, elderly and immunocompromised patients, including those with HIV, patients with poorly controlled diabetes mellitus or chronic kidney disease or those receiving immunosuppressant treatments, are generally more susceptible to the

(c) The Author(s). 2018 Open Access This article is distributed under the terms of the Creative Commons Attribution 4.0 International License (http://creativecommons.org/licenses/by/4.0/), which permits unrestricted use, distribution, and reproduction in any medium, provided you give appropriate credit to the original author(s) and the source, provide a link to the Creative Commons license, and indicate if changes were made. The Creative Commons Public Domain Dedication waiver (http://creativecommons.org/publicdomain/zero/1.0/) applies to the data made available in this article, unless otherwise stated. 
disease [4]. Healthcare workers who are exposed to TB also have higher risks of being infected [5].

Due to the side effects of the drugs [6,7], poor medication compliance and the problem of development of drug-resistant TB, global investment in TB research is needed [8]. Among the first-line anti-TB drugs, side effects of hepatotoxicity have been reported to be associated with isoniazid, rifampin and pyrazinamide; cutaneous reactions with isoniazid, pyrazinamide and ethambutol; gastrointestinal intolerance with rifampin; retrobulbar neuritis with ethambutol; and ototoxicity with streptomycin [9-11]. Long-term respiratory symptoms and impairment of pulmonary function affect patients' quality of life after completing TB treatment. The respiratory comorbidities were identified in more than half of microbiologically cured TB patients [12]. Despite successful treatment of TB, residual impairments persist, and chronic complications or sequelaes can arise from structural or vascular alterations at disease sites $[13,14]$. Many patients with chronic illness may seek complementary therapies [15], especially Chinese Medicine (CM) in Asian countries [16, 17]. CM, which originated in ancient China, is defined as comprehensive healthcare skills and practice for the maintenance of health and treatment of disease based on the beliefs of holism [18] and experiences of pattern identification/ syndrome differentiation handed down from generation to generation [19]. In Taiwan, CM is important and popular among different categories of complementary therapies, and is regarded as one of the mainstream therapies with coverage of the National Health Insurance (NHI) program [17]. "CM" usually refers to the overall treatment modalities, and services of CM covered by the NHI program, which includes Chinese medicines, acupuncture, moxibustion and Chinese traumatology therapy [20]. "Chinese medicines" usually refers to herbs or herbal products which can be classified into single-herb products and herbal formulas (multi-herb products) [21]. In Taiwan, herbal products (concentrated scientific herbal granules) covered by the NHI program are manufactured by GMP-certified pharmaceutical companies [22].

The ancient CM literature did not use the word "TB", but rather considered this pulmonary condition to be related to a syndrome of lung consumption characterized by cough, hemoptysis, tidal fever, night sweats and emaciation due to consumptive or exhaustive overstrain. It was reported that Chinese medicines combined with conventional medicine has beneficial effects for inhibiting Mycobacterium, strengthening the immune system of the body, enhancing the effect of anti-TB drugs, reducing drug resistance, and being relatively safe [23-25]. While several studies have investigated the use of CM in the care of TB patients [23], there is a lack of this kind of survey on the use of CM among patients with a medical history of TB and complicated with long-term respiratory discomforts.
We intended to investigate the utilization of $\mathrm{CM}$ among patients with a medical history of TB complicated with long-term respiratory discomforts in this study. The results of this study will be useful for future clinical trials and pharmacological investigations regarding efficacy and safety.

\section{Materials and methods}

\section{Data sources}

The NHI program was established in Taiwan in 1995. The program was highly representative of samples of Taiwan's general population because the reimbursement policy is universal and mandatory. The coverage rate of this compulsory health insurance program reached almost 95\% in 1997 [26] and 99.4\% at the end of 2010 in Taiwan [27]. CM services that are reimbursed in the NHI program include Chinese medicines, acupuncture, moxibustion, and Chinese traumatology therapy [28]. The National Health Insurance Administration provided the registration files and original claims data to the $\mathrm{Na}$ tional Health Research Institutes, which established and managed the National Health Insurance Research Database (NHIRD) for research purposes. This study was based in part on data from the NHIRD. It contains data comprising demographic characteristics, medical care facilities, outpatient and inpatient visits, visit dates, diagnostic codes, management, prescriptions and medical expenditures. We acquired a randomly selected sample consisting of one million individuals (Longitudinal Health Insurance Database 2000; LHID 2000) from the NHIRD managed and released by the National Health Research Institutes, Taiwan. The diagnostic codes were from the International Classification of Diseases, Ninth Revision, Clinical Modification (ICD-9-CM) formats.

\section{Ethical consideration}

This study followed the ethical standards of the responsible committee and with the Helsinki Declaration of 1964 and later versions. All the datasets were de-identified and encrypted before released by the National Health Research Institutes, Taiwan. All of the individuals or care providers could not be identified in the database. Patient consent was exempted for the total anonymity of all research data in this study. Therefore, the Research Ethics Committee of China Medical University and Hospital approved this study and waived the requirement for informed consent (CMUH104-REC2-115).

\section{Study population}

Patients newly diagnosed with TB (ICD-9-CM codes 010-018 and A02) $(n=36,660)$ were selected from the database. To avoid the inclusion of patients who did not have the disease, we set the inclusion criteria to allow only patients who were newly diagnosed with TB from January 1, 1997, to December 31, 2010, with clinical 
records from at least 2 months and treatment with first-line TB medication prescriptions (isoniazid, rifampin, pyrazinamide \& ethambutol) $(n=8306)$. Because the prevalence and etiology of TB infections in children were different, and the medical services-seeking behaviors among children or adolescents were mainly dominated by their parents, children with TB infections were not included in our objectives. Therefore, we further excluded patients who were less than 18 years of age or who had missing information related to birth and sex $(n=401)$. A total of 7905 TB patients were then included. Therefore the sample inclusion criteria is as following: (1) TB diagnosed between January 1, 1997 and December 31, 2010. (2) 18 years old or over. (3) Clinical records for at least 2 months with complete demographic information (4) Record of treatment with first-line TB medication prescriptions. CM users were defined as those who had visited $\mathrm{CM}$ clinics and had CM outpatient clinical records of TB or respiratory diseases (ICD-9-CM codes 786.x or 460-519). Non-CM users were defined as those who never visited $\mathrm{CM}$ clinics after the initial diagnosis of TB. To investigate TB patients complicated with long- term respiratory discomforts, we further excluded those clinical visits with a non-TB or respiratory diagnosis $(n=3980)$. Finally, a total of $3925 \mathrm{~TB}$ patients were then categorized into CM users $(n=2051)$ and non-CM users $(n=1874)$ (Fig. 1). Taiwan is a country with a population of 23 million. Taiwan has a geographical area of $36,000 \mathrm{~km}^{2}$. There were 23 cities and 359 townships in Taiwan and many of its residents live within urban cities. The residential areas of 23 cities in Taiwan were classified into 4 levels of urbanization based on the population density (people $/ \mathrm{km}^{2}$ ), the ratio of the population with varying educational levels, and the number of physicians per 100,000 people. Level 1 represents the highest urbanized level, while 4 represents the lowest level. Levels 1 and 2 of this urbanization were defined as urban areas, while levels 3 and 4 were classified as rural areas [29].

\section{Prescription of Chinese medicines}

We listed the herbal formulas by their phonetic pin-yin name and single herbs by their phonetic pin-yin name, Chinese material medica name and plant name. Indications for the herbal formulas and single herbs were based on CM theory [30, 31]. Full botanical names were in accordance with the International Plant Names List (IPNI; http://www.ipni.org) and The Plant List (http://

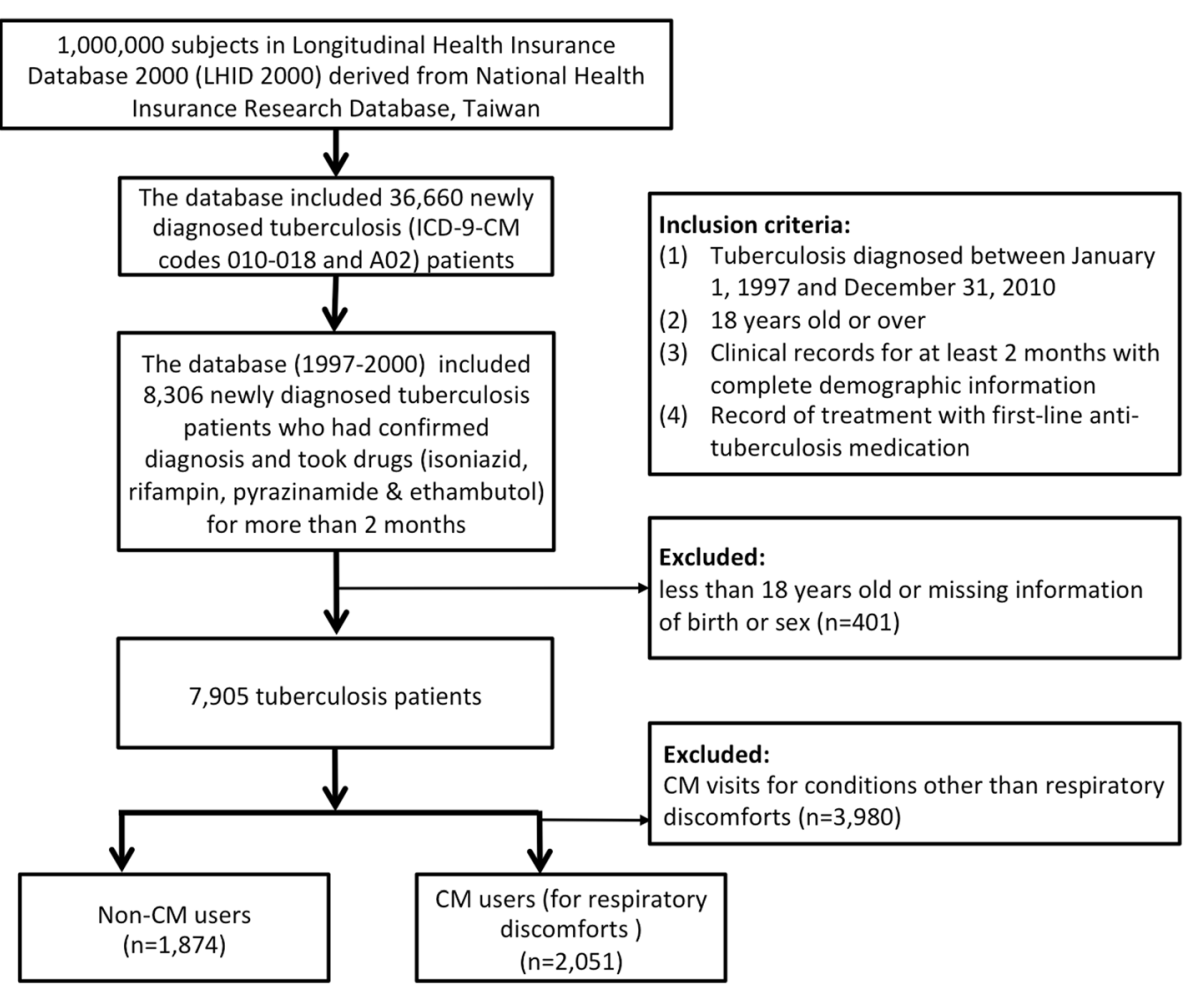

Fig. 1 Recruitment flow chart of subjects with tuberculosis (TB) from one million randomly selected samples in the National Health Insurance Research Database (NHIRD) from 1997 to 2000 in Taiwan. The inclusion criteria of patients ( $n=7905)$ with history of TB (ICD-9-CM codes 010-018 and A02) were: (1) TB diagnosed between January 1, 1997 and December 31, 2010 (2) 18 years old or over (3) Clinical records for at least 2 months with complete demographic information (4) Record of treatment with first-line TB medication prescriptions. CM users for conditions other than respiratory discomforts $(n=3980)$ were excluded. Finally, a total of 3925 TB patients were categorized as: CM users for respiratory discomforts $(n=2051)$ and non-CM users ( $n=1874)$ 
www.theplantlist.org/) [32]. An open-sourced freeware Node XL (http://nodexl.codeplex.com/) was utilized to identify the core patterns of prescriptions for TB patients, and the most common co-prescribed Chinese medicines were demonstrated in this network analysis. As previously described [33], the larger of the spots with thicker-line widths indicated significant prescription patterns and counts of close connections between formulas and herbs in the network figure.

\section{Statistical analysis}

All statistical analyses were performed using SAS software, version 9.4 (SAS Institute Inc., Cary, NC, USA). Univariate analysis was utilized to compare the CM users with the non-CM users. Data analysis comprised descriptive statistics, including the frequency of herbal prescriptions, patient demographic characteristics, indications for the TCM prescription, and the most frequently prescribed herbal formulas and herbs for treating TB. The chi-square test was used to examine the relationships between the categorical variables and to examine the differences between $\mathrm{CM}$ users and non-CM users. A $P$-value of $<0.05$ was considered statistically significant.

\section{Results}

In this study, we identified $52.25 \%(n=2051)$ patients with a medical history of TB who had visited CM clinics and had CM outpatient clinical records of TB or respiratory diseases (Fig. 1). Those who were female, younger (18-39 y/o), and lived in higher urbanized areas (levels 1 and 2) were relatively more likely to use CM. The median duration between newly diagnosed TB and the first CM consultation was 1068 days (Table 1).

Regarding the treatment modes employed among the CM users, approximately $95 \%$ of CM users $(n=1944)$ received only Chinese medicines, $5.2 \%$ of patients received combined treatment with both Chinese medicines and acupuncture, and the remaining $0.2 \%$ of patients received only acupuncture treatments. Among all CM users (patients with a medical history of TB who had visited $\mathrm{CM}$ clinics and had $\mathrm{CM}$ outpatient clinical records of TB or respiratory diseases; $n=2051$ ), $90.15 \%$ visited CM clinics $1-3$ times annually, while $4.58 \%$ patients consulted CM doctors more than 6 times/year (Table 2).

The frequency distribution of disease categories related to respiratory discomforts and comorbidities that patients who had been diagnosed TB was analyzed (Table 3). Compared to the non-CM users, the frequencies of outpatient visits among TB patients who used CM were statistically higher across almost all discomforts and comorbidities, except swelling, mass, and lump in chest (Table 3). More than $70 \%$ of TB patients used CM because of acute respiratory infections, cough, chronic obstructive pulmonary disease and allied conditions, and other diseases of upper respiratory tract. These results could indicate the comorbidities and complications of TB and may serve as an explanation for why TB patients complicated with long-term respiratory discomforts turn to $\mathrm{CM}$ doctors for help (Table 3). We further investigated the prescription pattern of Chinese medicines and identified the ten most commonly prescribed formulas and single herbs. The ten most commonly prescribed single herbs and herbal formulas were analyzed and are listed in Tables 4 and 5, respectively. The most commonly prescribed herbal formulas and single herb were Xiao-Qing-Long-Tang, and Radix Platycodonis (Jie-Geng), respectively.

We further conducted a network analysis and found that the core patterns of herbal formulas and herbs prescribed for TB patients consisted of Ma-Xing-Gan-Shi-Tang, Bulbus Fritillariae Thunbergii (Bei-Mu), Radix Platycodonis (Jie-Geng), and Semen Armeniacae (Xing-Ren) (Fig. 2). We further summarized these findings in Fig. 3.

\section{Discussion}

In this study, we first observed that the median duration between newly diagnosed TB and the first CM consultation was 1068 days. Many TB patients complicated with long-term respiratory discomforts sought $\mathrm{CM}$ services. We then determined that Ma-Xing-Gan-Shi-Tang, Bulbus Fritillariae Thunbergii (Bei-Mu), Radix Platycodonis (Jie-Geng), and Semen Armeniacae (Xing-Ren) were the core prescriptions for patients with $\mathrm{TB}$ in Taiwan. To the best of our knowledge, this report describes the first nationwide population-based cohort study investigating $\mathrm{CM}$ utilization patterns among $\mathrm{TB}$ patients complicated with long-term respiratory discomforts. However, whether CM combined with modern conventional medicine has beneficial effects or risks requires further investigation.

The phenomenon that female patients with TB were relatively more likely to seek $\mathrm{CM}$ services is comparable to a previous Taiwanese survey demonstrating that women had a greater tendency to seek CM consultations [34]. It has been reported that women are more susceptible to side effects from anti-TB drugs due to higher CYP3A activity [11, 35], although men are more susceptible to TB than women [36]. Women may exhibit more help-seeking behaviors than men and are more likely to seek complementary therapies when they suffer from chronic or catastrophic illness [37, 38].

We also observed that younger patients (18-39 y/o) and those who lived in more urbanized areas were relatively more likely to choose CM, in accordance with previous studies [38, 39]. Patients younger than 40 years old are likely to be more aware of alternative therapeutic choices. A previous report had shown that women, people with high socioeconomic status, higher education levels, self-perceived poor health status, and people who 
Table 1 Demographic characteristics of the patients with tuberculosis in Taiwan

\begin{tabular}{|c|c|c|c|c|c|}
\hline \multirow[t]{3}{*}{ Variable } & \multirow{2}{*}{\multicolumn{2}{|c|}{$\begin{array}{l}\text { Non-CM users } \\
\mathrm{n}=1874(44.753 \%)\end{array}$}} & \multirow{2}{*}{\multicolumn{2}{|c|}{$\begin{array}{l}\text { CM users }^{\dagger} \\
\mathrm{n}=2051(52.25 \%)\end{array}$}} & \multirow[t]{3}{*}{$P$-value } \\
\hline & & & & & \\
\hline & $n$ & $\%$ & $n$ & $\%$ & \\
\hline Sex & & & & & $<.0001^{*}$ \\
\hline Female & 354 & 18.89 & 861 & 41.98 & \\
\hline Male & 1520 & 81.11 & 1190 & 58.02 & \\
\hline Age at baseline (year) & & & & & $<.0001^{*}$ \\
\hline $18-29$ & 100 & 5.34 & 293 & 14.29 & \\
\hline $30-39$ & 127 & 6.78 & 242 & 11.8 & \\
\hline$\geq 40$ & 1647 & 87.89 & 1516 & 73.92 & \\
\hline Mean (SD) & \multicolumn{2}{|c|}{$64.06(17.68)$} & \multicolumn{2}{|c|}{$53.25(18.03)$} & $<.0001 \neq$ \\
\hline Urbanization & & & & & $<.0001^{*}$ \\
\hline 1 (highest) & 408 & 21.77 & 529 & 25.8 & \\
\hline 2 & 431 & 23 & 607 & 29.61 & \\
\hline 3 & 327 & 17.45 & 345 & 16.83 & \\
\hline 4 & 354 & 18.89 & 306 & 14.93 & \\
\hline $5+$ (lowest) & 354 & 18.89 & 263 & 12.83 & \\
\hline \multicolumn{6}{|l|}{ Drug used } \\
\hline rifampin & 1819 & 97.07 & 2005 & 97.76 & $0.1714^{*}$ \\
\hline ethambutol & 1796 & 95.84 & 1985 & 96.78 & $0.116^{*}$ \\
\hline isoniazid & 1802 & 96.16 & 1972 & 96.15 & $0.9874^{*}$ \\
\hline pyrazinamide & 1581 & 84.36 & 1798 & 87.66 & $0.0028^{*}$ \\
\hline levofloxacin & 456 & 24.33 & 536 & 26.13 & $0.1948^{*}$ \\
\hline streptomycin & 131 & 6.99 & 113 & 5.51 & $0.055^{*}$ \\
\hline kanamycin & 73 & 3.9 & 81 & 3.95 & $0.9308^{*}$ \\
\hline prothionamide & 18 & 0.96 & 24 & 1.17 & $0.5237^{*}$ \\
\hline cyclosporine & 2 & 0.11 & 5 & 0.24 & $0.456 \$$ \\
\hline moxifloxacin & 0 & 0 & 1 & 0.05 & - \\
\hline \multicolumn{3}{|l|}{ Interval between the onset oftuberculosis and the first CM consultation, days(median) } & \multicolumn{2}{|c|}{1395 (1068) } & - \\
\hline
\end{tabular}

\# t-test; * chi-square; \$ Fisher's exact test

Abbreviation: $S D$ standard deviation, $C M$ Chinese Medicine

† CM users referred to patients with history of TB who had visited CM clinics and had CM outpatient clinical records of tuberculosis or respiratory diseases

exercise regularly tend to visit CM services more often in Taiwan [40]. Patients who reside in more urbanized areas usually have a higher socioeconomic status and fewer barriers to accessing medical services [41, 42]. The time and money spent on transportation may also lead to a significant inequality in contact with the health system [43-45]. Therefore, people who live in urbanized areas have a greater tendency to use CM services.

In Taiwan, patients with a diagnosis of $\mathrm{TB}$ are required to receive pharmacological therapy to control TB infection

Table 2 Distribution of CMs according to type of CM treatment received in patients with tuberculosis, stratified by the number of outpatient visits

\begin{tabular}{lcllc}
\hline $\begin{array}{l}\text { Number of CM } \\
\text { visits (times/per year) }\end{array}$ & $\begin{array}{l}\text { Only Chinese medicines } \\
n=1944(94.78 \%)\end{array}$ & $\begin{array}{l}\text { Only acupuncture } \\
n=4(0.20 \%)\end{array}$ & $\begin{array}{l}\text { Combination of both treatments } \\
n=103(5.02 \%)\end{array}$ & $\begin{array}{l}\text { Total } n=2051 \\
(100 \%)\end{array}$ \\
\hline $1-3$ & $\mathrm{n}(\%)$ & $\mathrm{n}(\%)$ & $82(76.61)$ & $\mathrm{n}(\%)$ \\
$4-6$ & $1763(90.69)$ & $4(100)$ & $12(11.65)$ & $1049(90.15)$ \\
$>6$ & $96(4.94)$ & 0 & $9(8.74)$ & $94(4.58)$ \\
\hline
\end{tabular}

Abbreviation: CM Chinese Medicine 
Table 3 Frequency of different diseases related to symptoms of respiratory discomforts or complications in tuberculosis patients

\begin{tabular}{|c|c|c|c|c|c|}
\hline \multirow[t]{2}{*}{ Disease (ICD-9-CM) } & \multicolumn{2}{|c|}{ Non-CM users } & \multicolumn{2}{|c|}{$\mathrm{CM}$ users $^{\dagger}$} & \multirow[t]{2}{*}{$P$ value } \\
\hline & $\mathrm{n}$ & $\%$ & $\mathrm{n}$ & $\%$ & \\
\hline Acute respiratory infections (460-466) & 1405 & 74.97 & 2022 & 98.59 & $<.0001$ \\
\hline Other diseases of upper respiratory tract (470-478) & 564 & 30.1 & 1544 & 75.28 & $<.0001$ \\
\hline Pneumonia and influenza (480-488) & 1156 & 61.69 & 1393 & 67.92 & $<.0001$ \\
\hline Chronic obstructive pulmonary disease and allied conditions (490-496) & 1307 & 69.74 & 1663 & 81.08 & $<.0001$ \\
\hline Pneumoconioses and other lung diseases due to external agents (500-508) & 183 & 9.77 & 146 & 7.12 & 0.0028 \\
\hline Other diseases of respiratory system (510-519) & 952 & 50.8 & 853 & 41.59 & $<.0001$ \\
\hline Dyspnea and respiratory abnormalities (786.0) & 313 & 16.7 & 488 & 23.79 & $<.0001$ \\
\hline Stridor (786.1) & 25 & 1.33 & 124 & 6.05 & $<.0001$ \\
\hline Cough (786.2) & 475 & 25.35 & 1519 & 74.06 & $<.0001$ \\
\hline Hemoptysis (786.3) & 211 & 11.26 & 375 & 18.28 & $<.0001$ \\
\hline Abnormal sputum (786.4) & 16 & 0.85 & 49 & 2.39 & 0.0002 \\
\hline Chest pain (786.5) & 375 & 20.01 & 930 & 45.34 & $<.0001$ \\
\hline Swelling, mass, or lump in chest (786.6) & 32 & 1.71 & 50 & 2.44 & 0.1101 \\
\hline Hiccough (786.8) & 9 & 0.48 & 40 & 1.95 & $<.0001$ \\
\hline Other symptoms involving respiratory system and chest (786.9) & 23 & 1.23 & 52 & 2.54 & 0.0028 \\
\hline
\end{tabular}

* chi-square test

Abbreviation: SD standard deviation, $C M$, Chinese Medicine

TCM users referred to patients with history of TB who had visited CM clinics and had CM outpatient clinical records of tuberculosis or respiratory diseases

with monitoring by local health officers based on the government's health policy [46]. The included patients who had been diagnosed with TB should have received standard anti-TB treatment. In Taiwan, recommended standard treatment for adult respiratory $\mathrm{TB}$ consists of a regimen of isoniazid, rifampicin, pyrazinamide, and ethambutol for 2 months, followed by isoniazid, rifampicin, and ethambutol for 4 months with monitoring by local health officers according to governmental policy [46]. The total treatment course takes approximately 6 months in newly diagnosed patients [46]. The median duration between newly diagnosed TB and the first $\mathrm{CM}$ consultation was 1068 days. During the follow-up period, patients might seek CM consultation due to symptoms of respiratory discomfort or complications. In addition to respiratory illness, they may also experience other symptoms related to the anti-TB

Table 4 Ten most commonly prescribed herbs for patients with tuberculosis

\begin{tabular}{|c|c|c|c|c|}
\hline Pin-yin name & $\begin{array}{l}\text { Chinese Materia } \\
\text { Medica name }\end{array}$ & Botanical name & Indication & $\begin{array}{l}\text { Number of } \\
\text { person-days }\end{array}$ \\
\hline Jie-Geng & Radix Platycodonis & Platycodon grandiflorus (Jacq.) A.DC & Cough, large amount of sputum, sore throat & 18,779 \\
\hline Bei-Mu & Bulbus Fritillariae Thunbergii & Fritillaria thunbergii Miq. & $\begin{array}{l}\text { Lung heat with thick phlegm, cough due } \\
\text { to yin deficiency }\end{array}$ & 18,460 \\
\hline Xing-Ren & Semen Armeniacae & Prunus armeniaca L.var. ansu Max & $\begin{array}{l}\text { Cough with phlegm, cough in older people } \\
\text { or weaker bodies }\end{array}$ & 14,610 \\
\hline Yu-Xing- Cao & Herba Houttuyniae & Houttuynia cordata Thunb. & Fever, Inflammation of the respiratory tract & 8978 \\
\hline Gua-Lou- Ren & Semen Trichosanthis & Trichosanthes kirilowii Maxim & Hot cough with sticky phlegm & 8905 \\
\hline Gan-Cao & Radix Glycyrrhizae & Glycyrrhiza uralensis Fisch & $\begin{array}{l}\text { Lung TB, cough with abundance of phlegm, } \\
\text { tired and lack of strength, palpitation and } \\
\text { short of breath }\end{array}$ & 8054 \\
\hline Huang-Qin & Radix Scutellariae & Scutellaria baicalensis Georgi & $\begin{array}{l}\text { Cough due to heat syndromes, infection, } \\
\text { and hemoptysis }\end{array}$ & 7991 \\
\hline Mai-Men-Dong & Radix Ophiopogonis & Ophiopogon japonicus (Thunb.) Ker Gawl. & $\begin{array}{l}\text { Cough, weakness, consumption, short } \\
\text { of breath, heat from yin deficiency }\end{array}$ & 6934 \\
\hline Dan-Shen & Radix Salviae Miltiorrhizae & Salvia miltiorrhiza Bge. & $\begin{array}{l}\text { Restlessness, insomnia, irritability, blood } \\
\text { deficiency and blood stasis }\end{array}$ & 6508 \\
\hline Wu-Wei- Zi & Fructus Schisandrae & Schisandra chinensis (Turcz.) Baill. & $\begin{array}{l}\text { Wheezy cough, palpitations, and thirst due } \\
\text { to yin deficiency }\end{array}$ & 6154 \\
\hline
\end{tabular}


Table 5 Ten most commonly prescribed formulas for patients with tuberculosis

\begin{tabular}{|c|c|c|c|c|c|}
\hline \multirow[t]{2}{*}{ Pin-yin name } & \multicolumn{3}{|l|}{ Constitutions } & \multirow[t]{2}{*}{ Indications in TCM } & \multirow{2}{*}{$\begin{array}{l}\text { Number of } \\
\text { person- days }\end{array}$} \\
\hline & Pin-yin name & Chinese Materia Medica name & Botanical name & & \\
\hline \multirow[t]{8}{*}{ Xiao-Qing-Long-Tang } & Ma-Huang & Herba Ephedrae & Ephedra sinica Stapf. & \multirow{8}{*}{$\begin{array}{l}\text { Coughing and } \\
\text { wheezing with } \\
\text { copious, white, } \\
\text { stringy sputum } \\
\text { that is difficult to } \\
\text { expectorate, stifling } \\
\text { sensation in the } \\
\text { chest, chronic } \\
\text { water metabolism } \\
\text { problems and thin } \\
\text { mucus associated } \\
\text { with weakness } \\
\text { of lung }\end{array}$} & \multirow[t]{8}{*}{16,050} \\
\hline & Gui-Zhi & Ramulus Cinnamomi & $\begin{array}{l}\text { Cinnamomum } \\
\text { cassia Blume }\end{array}$ & & \\
\hline & Gan-Jiang & Rhizoma Zingiberis & Zingiber officinale Rosc & & \\
\hline & Xi-Xin & Herba Asari & $\begin{array}{l}\text { Asarum heterotropoides } \\
\text { F. Schmidt }\end{array}$ & & \\
\hline & Wu-Wei-Zi & Fructus Schisandrae & $\begin{array}{l}\text { Schisandra chinensis } \\
\text { (Turcz.) Baill. }\end{array}$ & & \\
\hline & Bai-Shao & Radix Paeoniae Alba & Paeonia lactiflora Pall. & & \\
\hline & Ban-Xia & Rhizoma Pinelliae & $\begin{array}{l}\text { Pinellia ternate } \\
\text { (Thunb.) Makino }\end{array}$ & & \\
\hline & Gan-Cao & Radix Glycyrrhizae & Glycyrrhiza uralensis Fisch & & \\
\hline \multirow[t]{10}{*}{ Xin-Yi-Qing-Fei-Tang } & $X i n-Y i$ & Magnoliae Flos & Magnolia biondii Pamp. & \multirow{10}{*}{$\begin{array}{l}\text { Lung-heat cough } \\
\text { with yellow phlegm, } \\
\text { and accumulation } \\
\text { of lung heat with } \\
\text { nasal congestion }\end{array}$} & \multirow[t]{10}{*}{15,035} \\
\hline & Huang-Qin & Radix Scutellariae & Scutellaria baicalensis Georgi & & \\
\hline & Mai-Men-Dong & Radix Ophiopogonis & $\begin{array}{l}\text { Ophiopogon japonicus } \\
\text { (Thunb.) Ker Gawl. }\end{array}$ & & \\
\hline & Zhi-Zi & Fructus Gardeniae & Gardenia jasminoides J.Ellis & & \\
\hline & Shi- Gao & Gypsum fibrosum & Hydrous Calcium Sulfate & & \\
\hline & Zhi-Mu & Rhizoma Anemarrhenae & $\begin{array}{l}\text { Anemarrhena asphodeloides } \\
\text { Bunge }\end{array}$ & & \\
\hline & Sheng-Ma & Radix Cimicifugae & $\begin{array}{l}\text { Cimicifuga foetida } \mathrm{L} \text {. } \\
\text { var., intermedia, Regel }\end{array}$ & & \\
\hline & Bai-He & Bulbus Lilii & $\begin{array}{l}\text { Lilium brownii F. E. } \\
\text { Br.ex Meillez }\end{array}$ & & \\
\hline & $\mathrm{Pi}-\mathrm{Pa}-\mathrm{Ye}$ & Folium Eriobotryae & $\begin{array}{l}\text { Eriobotrya japonica } \\
\text { (Thunb) Lindl }\end{array}$ & & \\
\hline & Gan-Cao & Radix Glycyrrhizae & Glycyrrhiza uralensis Fisch & & \\
\hline \multirow[t]{4}{*}{ Ma-Xing-Gan-Shi-Tang } & Ma-Huang & Herba Ephedrae & Ephedra sinica Stapf. & \multirow{4}{*}{$\begin{array}{l}\text { Fever with thirst, } \\
\text { wheezing, coughing, } \\
\text { labored breathing } \\
\text { caused by heat } \\
\text { lodged in the lungs } \\
\text { where it obstructs } \\
\text { the flow of qi }\end{array}$} & \multirow[t]{4}{*}{14,185} \\
\hline & Xing-Ren & Semen Armeniacae & $\begin{array}{l}\text { Prunus armeniaca } \\
\text { L.var. ansu Maxim }\end{array}$ & & \\
\hline & Shi- Gao & Gypsum fibrosum & Hydrous Calcium Sulfate & & \\
\hline & Gan-Cao & Radix Glycyrrhizae & Glycyrrhiza uralensis Fisch & & \\
\hline \multirow[t]{9}{*}{ Ding-Chuan-Tang } & Ma-Huang & Herba Ephedrae & Ephedra sinica Stapf. & \multirow{9}{*}{$\begin{array}{l}\text { Coughing and } \\
\text { wheezing with } \\
\text { thick, yellow sputum } \\
\text { that is difficult to } \\
\text { expectorate. Fever } \\
\text { and labored } \\
\text { breathing caused by } \\
\text { the disrupted flow } \\
\text { of lung qi that } \\
\text { transforms into heat }\end{array}$} & \multirow[t]{9}{*}{10,664} \\
\hline & Xing-Ren & Semen Armeniacae & $\begin{array}{l}\text { Prunus armeniaca } \\
\text { L.var. ansu Maxim }\end{array}$ & & \\
\hline & Ban-Xia & Rhizoma Pinelliae & $\begin{array}{l}\text { Pinellia ternate } \\
\text { (Thunb.) Makino }\end{array}$ & & \\
\hline & Huang-Qin & Radix Scutellariae & Scutellaria baicalensis Georgi & & \\
\hline & Sang-Bai-Pi & Cortex Mori & Morus alba L. & & \\
\hline & Bai-Guo & Semen Ginkgo & Ginkgo biloba L. & & \\
\hline & Su-Zi & Fructus Perillae & Perilla frutescens (L.) Britton. & & \\
\hline & $\begin{array}{l}\text { Kuan-Dong- } \\
\text { Hua }\end{array}$ & Flos Farfarae & Tussilago farfara $\mathrm{L}$. & & \\
\hline & Gan-Cao & Radix Glycyrrhizae & Glycyrrhiza uralensis Fisch & & \\
\hline \multirow[t]{3}{*}{ Yin-Qiao-San } & Jin-Yin-Hua & Flos Lonicerae & Lonicera japonica Thunb. & \multirow{3}{*}{$\begin{array}{l}\text { Fever, headache, } \\
\text { thirst, cough with } \\
\text { chills caused by } \\
\text { warm pathogens or } \\
\text { wind-heat entering }\end{array}$} & \multirow[t]{3}{*}{10,106} \\
\hline & Lian-Qiao & Fructus Forsythiae & $\begin{array}{l}\text { Forsythia suspensa } \\
\text { (Thunb.) Vahl }\end{array}$ & & \\
\hline & Jie-Geng & Radix Platycodonis & Platycodon grandiflorus (Jacq.) A.DC. & & \\
\hline
\end{tabular}


Table 5 Ten most commonly prescribed formulas for patients with tuberculosis (Continued)

\begin{tabular}{|c|c|c|c|c|c|}
\hline \multirow[t]{2}{*}{ Pin-yin name } & \multicolumn{3}{|l|}{ Constitutions } & \multirow[t]{2}{*}{ Indications in TCM } & \multirow{2}{*}{$\begin{array}{l}\text { Number of } \\
\text { person- days }\end{array}$} \\
\hline & Pin-yin name & Chinese Materia Medica name & Botanical name & & \\
\hline & Niu-Bang Zi & Fructus Arctii & Arctium lappa $\mathrm{L}$ & \multirow{7}{*}{$\begin{array}{l}\text { the body and } \\
\text { attacking the lungs }\end{array}$} & \\
\hline & Bo-He & Herba Menthae haplocalycis & Mentha haplocalyx Briq & & \\
\hline & Dan-Dou- Chi & Semen Sojae preparatum & Glycine max (L.) Merr. & & \\
\hline & Jing-Jie & Herba Schizonepetae & $\begin{array}{l}\text { Schizonepeta tenuifolia } \\
\text { (Benth.) }\end{array}$ & & \\
\hline & Dan-Zhu-Ye & Herba Lophatheri & Lophatherum gracile Brongn & & \\
\hline & Lu-Gen & Rhizoma Phragmitis recens & Phragmites communis Trin & & \\
\hline & Gan-Cao & Radix Glycyrrhizae & Glycyrrhiza uralensis Fisch & & \\
\hline \multirow[t]{10}{*}{ Bai-He-Gu Jin-Tang } & Shu-Di-Huang & Radix Rehmanniae & $\begin{array}{l}\text { Rehmannia glutinosa } \\
\text { Libosch }\end{array}$ & \multirow{10}{*}{$\begin{array}{l}\text { Coughing with } \\
\text { blood-streaked } \\
\text { sputum, wheezing, } \\
\text { dry and sore throat, } \\
\text { hot palms and soles, } \\
\text { night sweats due to } \\
\text { yin deficiency of lung } \\
\text { and kidney with heat } \\
\text { from yin deficiency }\end{array}$} & \multirow[t]{10}{*}{9735} \\
\hline & $\begin{array}{l}\text { Sheng-Di- } \\
\text { Huang }\end{array}$ & Radix Rehmanniae & $\begin{array}{l}\text { Rehmannia glutinosa } \\
\text { Libosch }\end{array}$ & & \\
\hline & Mai-Men-Dong & Radix Ophiopogonis & $\begin{array}{l}\text { Ophiopogon japonicus } \\
\text { (Thunb.) Ker Gawl. }\end{array}$ & & \\
\hline & Bai-He & Bulbus Lilii & $\begin{array}{l}\text { Lilium brownii F. E. } \\
\text { Br.ex Meillez }\end{array}$ & & \\
\hline & Bai-Shao & Radix Paeoniae Alba & Paeonia lactiflora Pall & & \\
\hline & Dang-Gui & Radix Angelicae Sinensis & Angelica sinensis (Oliv.) Diels & & \\
\hline & Bei-Mu & Bulbus Fritillariae Thunbergii & Fritillaria thunbergii Miq. & & \\
\hline & Jie-Geng & Radix Platycodonis & $\begin{array}{l}\text { Platycodon grandiflorus } \\
\text { (Jacq.) A.DC. }\end{array}$ & & \\
\hline & Xuan-Shen & Radix Scrophulariae & $\begin{array}{l}\text { Scrophularia ningpoensis } \\
\text { Hemsl. }\end{array}$ & & \\
\hline & Gan-Cao & Radix Glycyrrhizae & Glycyrrhiza uralensis Fisch & & \\
\hline \multirow[t]{12}{*}{ Xing-Su-Yin } & Zi-Su & Folium Perillae & Perilla frutescens (L.) Britton. & \multirow{12}{*}{$\begin{array}{l}\text { Wheezy cough, nasal } \\
\text { congestion, low fever, } \\
\text { headache due to a } \\
\text { wind-cold pathogen } \\
\text { attacking the lung }\end{array}$} & \multirow[t]{12}{*}{9343} \\
\hline & Xing-Ren & Semen Armeniacae & $\begin{array}{l}\text { Prunus armeniaca L.var. } \\
\text { ansu Maxim }\end{array}$ & & \\
\hline & Jie-Geng & Radix Platycodonis & Platycodon grandiflorus (Jacq.) A.DC. & & \\
\hline & Sang-Bai-Pi & Cortex Mori & Morus alba L. & & \\
\hline & Huang-Qin & Radix Scutellariae & Scutellaria baicalensis Georgi & & \\
\hline & Mai-Men-Dong & Radix Ophiopogonis & $\begin{array}{l}\text { Ophiopogon japonicus } \\
\text { (Thunb.) Ker Gawl. }\end{array}$ & & \\
\hline & Bei-Mu & Bulbus Fritillariae Thunbergii & Fritillaria thunbergii Miq. & & \\
\hline & Qian-Hu & Radix Peucedani & $\begin{array}{l}\text { Peucedanum decursivum } \\
\text { Maxim }\end{array}$ & & \\
\hline & Sheng-Jiang & Rhizoma Zingiberis & Zingiber officinale Rosc & & \\
\hline & Ju-Hong & $\begin{array}{l}\text { Citri Reticulatae } \\
\text { Pericarpium Rubrum }\end{array}$ & Citrus maxima (Burm.) Merr. & & \\
\hline & Zhi-Ke & Fructus Citri Aurantii & Citrus aurantium L. & & \\
\hline & Gan-Cao & Radix Glycyrrhizae & Glycyrrhiza uralensis Fisch & & \\
\hline \multirow[t]{5}{*}{ Zhi-Sou-San } & Jie-Geng & Radix Platycodonis & $\begin{array}{l}\text { Platycodon grandiflorus } \\
\text { (Jacq.) A.DC. }\end{array}$ & \multirow{5}{*}{$\begin{array}{l}\text { Coughing with slight } \\
\text { chills and fever, an } \\
\text { itchy throat, phlegm } \\
\text { that is difficult to } \\
\text { expectorate that } \\
\text { occurs in externally } \\
\text { contracted wind cold }\end{array}$} & \multirow[t]{5}{*}{9275} \\
\hline & Jing-Jie & Herba Schizonepetae & $\begin{array}{l}\text { Schizonepeta tenuifolia } \\
\text { (Benth.) }\end{array}$ & & \\
\hline & Zi-Wan & Asteris Radix & Aster tataricus L. f. & & \\
\hline & Bai-Bu & Radix stemonae & Stemona sessilifolia (Miq.) Miq. & & \\
\hline & Bai-Qian & Rhizoma Cynanchi Stauntonii & $\begin{array}{l}\text { Cynanchum stauntonii (Decne.) } \\
\text { Schltrex HLe }\end{array}$ & & \\
\hline
\end{tabular}


Table 5 Ten most commonly prescribed formulas for patients with tuberculosis (Continued)

\begin{tabular}{|c|c|c|c|c|c|}
\hline \multirow[t]{2}{*}{ Pin-yin name } & \multicolumn{3}{|l|}{ Constitutions } & \multirow[t]{2}{*}{ Indications in TCM } & \multirow{2}{*}{$\begin{array}{l}\text { Number of } \\
\text { person- days }\end{array}$} \\
\hline & Pin-yin name & Chinese Materia Medica name & Botanical name & & \\
\hline & Chen-Pi & Pericarpium Citri Reticulatae & Citrus reticulate Blanco & & \\
\hline & Gan-Cao & Radix Glycyrrhizae & Glycyrrhiza uralensis Fisch & & \\
\hline \multirow[t]{4}{*}{ Cang-Er-San } & Cang-Er-Zi & Xanthii Fructus & $\begin{array}{l}\text { Xanthium Sibiricum Patr. } \\
\text { ex Widder. }\end{array}$ & \multirow{4}{*}{$\begin{array}{l}\text { Nasal obstruction } \\
\text { with purulent nasal } \\
\text { discharge caused by } \\
\text { an external wind } \\
\text { pathogen obstructing } \\
\text { the protective qi, } \\
\text { which is governed by } \\
\text { lung, dizziness, } \\
\text { frontal headache }\end{array}$} & \multirow[t]{4}{*}{9248} \\
\hline & $X i n-Y i$ & Magnoliae Flos & Magnolia biondii Pamp. & & \\
\hline & Bo-He & Herba Menthae haplocalycis & Mentha haplocalyx Briq & & \\
\hline & Bai-Zhi & Radix Angelicae Dahuricae; & $\begin{array}{l}\text { Angelica dahurica (Hoffm.) } \\
\text { Benth. \& Hook.f. ex Franch. \& } \\
\text { Sav. }\end{array}$ & & \\
\hline \multirow[t]{9}{*}{ Qing-Zao-Jiu-Fei-Tang } & Sang-Ye & Folium Mori & Morus alba $\mathrm{L}$. & \multirow{9}{*}{$\begin{array}{l}\text { Wheezing, cough, dry, } \\
\text { parched throat, dry } \\
\text { nasal passages, } \\
\text { headache, fever, } \\
\text { irritability, thirst due } \\
\text { to invasion of external } \\
\text { warm-dryness attacking } \\
\text { the lungs and causing } \\
\text { damage to the lung } \\
\text { gi and yin }\end{array}$} & \multirow[t]{9}{*}{8795} \\
\hline & Shi-Gao & Gypsum fibrosum & Hydrous Calcium Sulfate & & \\
\hline & Gan-Cao & Radix Glycyrrhizae & Glycyrrhiza uralensis Fisch & & \\
\hline & Xing-Ren & Semen Armeniacae & $\begin{array}{l}\text { Prunus armeniaca L.var. } \\
\text { ansu Maxim }\end{array}$ & & \\
\hline & Mai-Men-Dong & Radix Ophiopogonis & $\begin{array}{l}\text { Ophiopogon japonicus } \\
\text { (Thunb.) Ker Gawl. }\end{array}$ & & \\
\hline & $\mathrm{Pi}-\mathrm{Pa}-\mathrm{Ye}$ & Folium Eriobotryae & $\begin{array}{l}\text { Eriobotrya japonica } \\
\text { (Thunb) Lindl }\end{array}$ & & \\
\hline & E-Jiao & Colla Corii Asini & Equus asinus L. & & \\
\hline & Ren-Shen & Radix Ginseng & Panax ginseng C. A. Mey & & \\
\hline & Hu-Ma-Ren & Semen Sesami Nigrum & Sesamum indicum L. & & \\
\hline
\end{tabular}

treatment, such as skin rash or drug fever, GI intolerance, nausea, vomiting, visual toxicity, hearing disturbances and arthralgia [9, 47-49]. The NHI insurance coverage for CM treatments may play a considerable role in patients' tendency to seek CM consultations. Medical services in both Western medicine and CM have been promoted due to the high coverage of the NHI program. Furthermore, the cost of co-payment for concentrated scientific herbal granules is approximately \$15 U.S. dollars per month under the NHI Program in Taiwan. The tendency of patients to seek CM may continue to increase since the co-payment for Chinese medicines is relatively low [50-53].

Among patients with TB, most CM users (94.78\%) received only Chinese medicines, which is consistent with previous studies investigating other respiratory diseases for which herbal products are frequently used as treatment in CM visits in Taiwan $[52,54]$. In patients with adult-onset asthma, $76.7 \%$ patients who visited CM physicians received Chinese medicines [54], and 97.1\% of the CM-treated rhinosinusitis subjects were prescribed Chinese medicines [52].

Regarding the disease categories of the CM users identified in Table 3, most of TB patients used CM because of acute respiratory infections, cough, chronic obstructive pulmonary disease and allied conditions, and other diseases of upper respiratory tract. It was reported that complications of TB include bacterial pneumonia, cor pulmonale, pneumothorax, and acute respiratory failure. Complications of TB could result in progression of functional pulmonary impairment and structural lung destructions [14, 55]. Patients with a prior TB diagnosis were more than twice as likely to have lung airflow obstruction [56]. Furthermore, the complications of respiratory diseases can be independent predictor of shorter survival among patients with TB [57]. TB patients might also turn to $\mathrm{CM}$ doctors in the hopes of relieving their symptoms and improving their health [58].

It must be noted that TB did not appear as a disease name in the records of the Chines medical literature. However, ancient people had observed the disease, and the symptoms and signs of TB and its related illness had been recorded and discussed in the Chinese medical literature. The prescriptions of $\mathrm{CM}$ were in accordance with the concepts of TB in CM, which were related to chronic cough with symptoms and signs of consumption or exhaustion. The prescriptions were prescribed after pattern identification and syndrome differentiation based on the theory of CM. Most of the prescriptions listed in Tables 4 and 5 are helpful for relieving symptoms caused by yin and qi deficiency of the lung with wind contraction, which corresponds to the respiratory symptoms related to long-term respiratory discomforts [19].

Among the single herbs identified in the core prescriptions, the herb Bulbus Fritillariae Thunbergii has been 


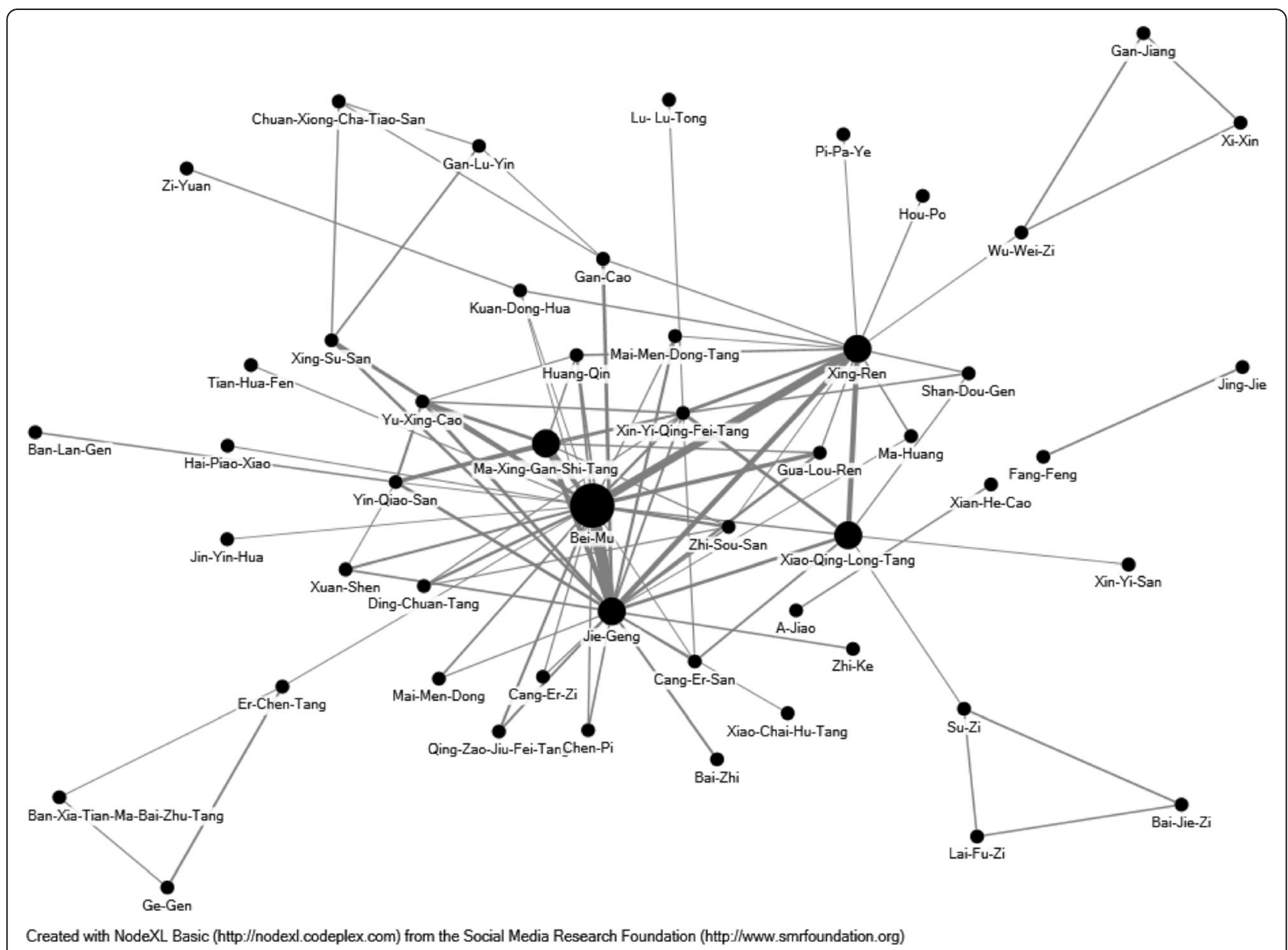

Fig. 2 The 100 most commonly prescribed herbal formulas and single herbs for patients with tuberculosis were assessed, and the core pattern of the prescriptions shows that Ma-Xing-Gan-Shi-Tang, Bei-Mu, Jie-Geng, and Xing-Ren were among the most frequently prescribed combinations

commonly used as an antitussive agent [59]. Its isosteroidal alkaloids have been reported to have tracheobronchial relaxant activity [60]. Radix Platycodonis has been used for bronchitis, tonsillitis, and laryngitis in ancient times. The pharmacological constituent Platycodin D in Radix Platycodonis has demonstrated anti-inflammatory, antitumor [61], antinociceptive and immunomodulatory activities. Immunostimulatory effects have been revealed via the suppression of IL-6 and TNF- $\alpha$ contents [62] and enhancement of the killing activities of natural killer cells [61]. Radix Platycodonis also inhibits OVA-induced airway inflammation and regulates the production and secretion of airway mucin [63]. Furthermore, Radix Platycodonis has often served as an expectorant in diverse inflammatory pulmonary diseases [64]. The other herb of the core prescriptions, Semen Armeniacae, has anti-inflammatory and analgesic effects by suppressing PGE2 and NO production [65]. The amygdalin found in it has been used as an antitussive, anti-asthmatic, anticancer agent $[66,67]$.
In addition to the above three herbs, other herbs have been reported to relieve respiratory symptoms or reduce inflammation. Herba Houttuyniae (Yu-Xing- Cao) has been reported to relieve fever, cough and asthma with anti-inflammatory [68] and antiviral activities [69]. This herb inhibits the production of pro-inflammatory cytokines via inhibition of the NF- $\kappa B$ signaling pathway in HMC-1 human mast cells [70]. Semen Trichosanthis (Gua-Lou- Ren) has anti-inflammatory, and antibacterial activities [71] and immunomodulatory effects [72]. Ophiopogonin-D in Radix Ophiopogonis (Mai-Men-Dong) may be beneficial for reducing the excitability of parasympathetic ganglionic neurons in the airways and cholinergic control of airway function, and its antitussive effects might be due to the activation of $\mathrm{K}+$ channels and hyperpolarization of paratracheal neurons [73]. Radix Salviae Miltiorrhizae (Dan-Shen) could be helpful for reducing lung fibrosis [74]. This species has been reported to exhibit anti-inflammatory and antioxidative effects [75]. Radix Scutellariae (Huang-Qin) has shown 


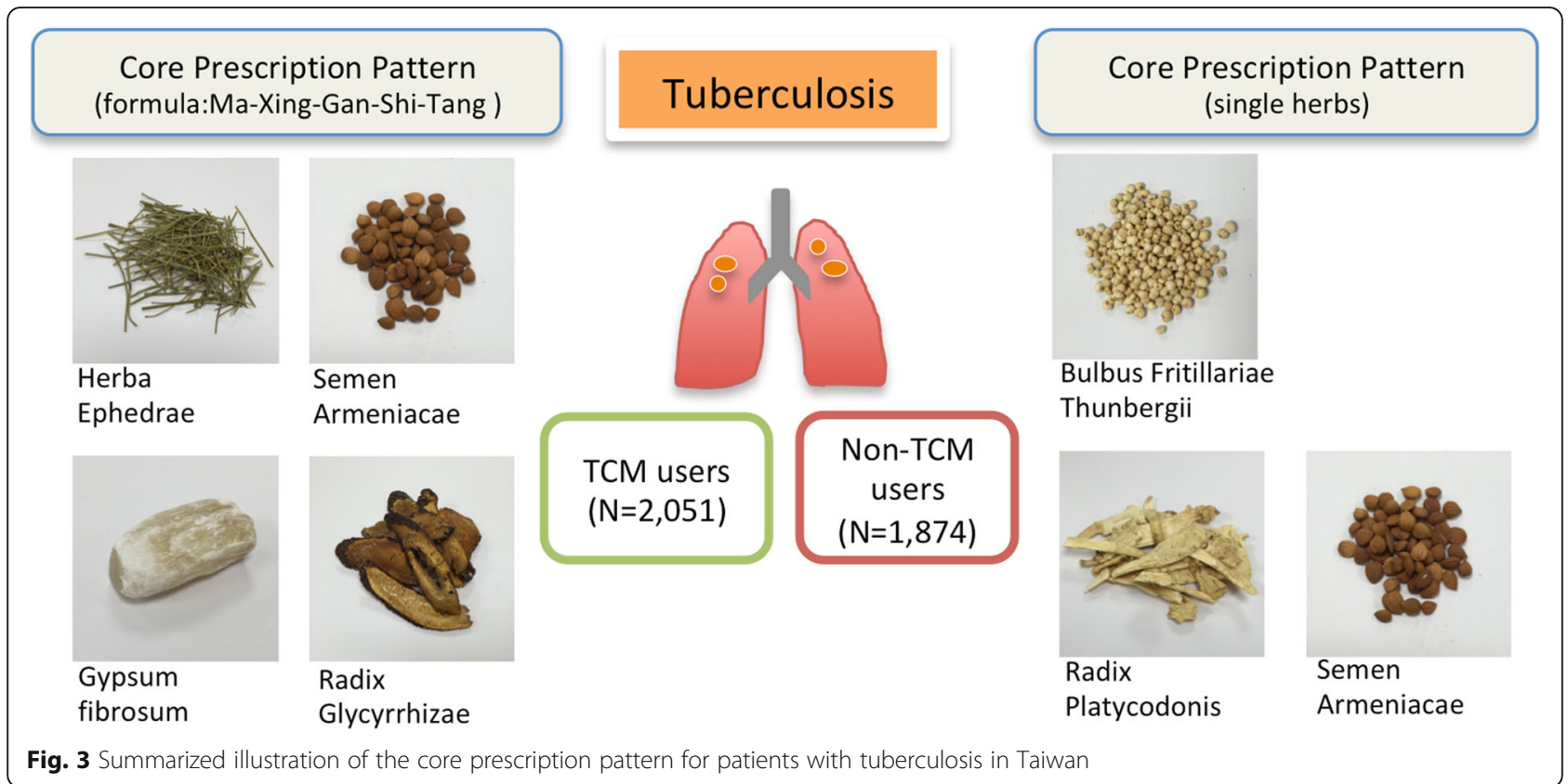

pharmacological effects that are anti-inflammatory, antitumor and hepato-protective. Additionally, these extracts have antioxidant, antibacterial and antiviral effects [76]. Research has indicated that a decoction containing Fructus Schisandrae (Wu-Wei-Zi) helps to prevent alveolitis and the development of pulmonary fibrosis [77]. Liver-protective effects of Fructus Schisandrae via the inhibitory effect of CYP-3A4 activity have been reported [78] and may also ameliorate the side effects of hepatitis resulting from the use of anti-TB medication.

Among the herbal formulas identified in this research, Ma-Xing-Gan-Shi-Tang is one of the core prescriptions. It has been used to treat asthma [79] and fever associated with pneumonia. It possesses anti-asthmatic, anti-pyretic, anti-inflammatory, and antitussive effects [80, 81]. Xiao-Qing-Long-Tang has anti-inflammatory, antiviral, and anti-allergy activities [82], and it can reduce inflammation in lung tissue via an anti-apoptotic effect and inhibition of cytokine release, as well as prevent pulmonary fibrosis [83-86]. Ding-Chuan-Tang has shown anti-asthmatic effects in clinical studies [87]. Its protective effects against lung injury in asthma occurs by suppressing the production of pro-inflammatory cytokines with significantly reduced inflammatory cell infiltration, goblet cell proliferation, collagen deposition, and damage in the bronchi and alveoli [88]. Xin-Yi-Qing-Fei-Tang has been commonly used for rhinitis $[51,52]$ and has been shown to reduce eosinophil, serum IgE and IL-4 levels [89]. Clinical studies have reported that Yi-Qiao-San may be helpful for patients with H1N1 influenza to reduce the duration of fever [90]. Lung cancer patients displayed better physical function, role function and cumulative survival after receiving combination treatments of a formula containing Bai-He-Gu Jin-Tang with Western medicine [91].

There are some limitations to our study. First, the herbal products purchased at the patients' own expense in addition to the NHI program products were not included in this study. However, because the co-payment of herbal products through the NHI program was much less than the market cost, the possibility of purchasing herbs outside the NHI program was relatively low. In addition, compliance to prescription regimens was difficult to measure. Finally, this study focuses on the utilization of $\mathrm{CM}$ in patients with TB. In the future, a high-quality randomized controlled clinical trial with imaging data and species cultures to determine the efficacy of Chinese medicines for TB is expected.

\section{Conclusions}

Our study found that many TB patients complicated with long-term respiratory discomforts sought CM services in Taiwan. Those who were 18-39 y/o, female, and who lived in urbanized areas tended to use CM. The prescription patterns identified in this study could be useful for future clinical studies or pharmacological investigations.

\footnotetext{
Abbreviations

CM: Chinese Medicine; ICD-9-CM: International Classification of Diseases, Ninth Revision, Clinical Modification; NHI: National Health Insurance; NHIRD: National Health Insurance Research Database; TB: Tuberculosis
}

\section{Acknowledgments}

This study was based in part on data from the National Health Insurance Research Database, provided by the National Health Insurance Administration, Ministry of Health and Welfare, and managed by National Health Research 
Institutes. The interpretation and conclusions contained herein do not represent those of the National Health Insurance Administration, Ministry of Health and Welfare, or National Health Research Institutes.

\section{Funding}

This work was financially supported by the Chinese Medicine Research Center, China Medical University, under the Featured Areas Research Center Program within the framework of the Higher Education Sprout Project by the Ministry of Education (MOE) in Taiwan (CMRC-CHM-2). This study was also supported in part by China Medical University Hospital (DMR-106183 and DMR-104-04) and the Taiwan Ministry of Health and Welfare Clinical Trial and Research Center of Excellence (MOHW107-TDU-B-212-123004) and Health and the welfare surcharge of tobacco products, China Medical University Hospital Cancer Research Center of Excellence (MOHW107-TDU-B212-114024), Taiwan. The funders had no role in the study design, data collection and analysis, decision to publish, or preparation of the manuscript.

\section{Availability of data and materials}

The datasets analyzed from NHIRD were provided by the National Health Insurance Administration and maintained by the National Health Research Institutes of Taiwan. The use of NHIRD is limited to research purposes only. Applicants must follow the Computer-Processed Personal Data Protection Law (http://www.winklerpartners.com/?p=987) and related regulations of the National Health Insurance Administration and National Health Research Institutes.

\section{Authors' contributions}

STY conceptualized the study. JHC performed the statistical analysis. STY, YRL, MYW, PSY and HRY contributed to the interpretation of the TCM data and pharmacological mechanisms. TCH contributed to the interpretation of Western medicine data. STY, YRL and HRY drafted the manuscript. STY and HRY finalized the manuscript. All authors read and approved the final manuscript.

\section{Ethics approval and consent to participate}

This study followed the ethical standards of the responsible committee and with the Helsinki Declaration of 1964 and later versions. All the datasets were de-identified and encrypted before released by the National Health Research Institutes, Taiwan. All of the individuals or care providers could not be identified in the database. Patient consent was exempted for the total anonymity of all research data in this study. Therefore, the Research Ethics Committee of China Medical University and Hospital approved this study and waived the requirement for informed consent (CMUH104-REC2-115).

\section{Consent for publication}

Not applicable.

\section{Competing interests}

The authors declare that they have no conflicts of interest to disclose.

\section{Publisher's Note}

Springer Nature remains neutral with regard to jurisdictional claims in published maps and institutional affiliations.

\section{Author details}

${ }^{1}$ Graduate Institute of Chinese Medicine, School of Chinese Medicine, College of Chinese Medicine, China Medical University, Taichung, Taiwan. ${ }^{2}$ Department of Radiology, China Medical University Hospital, Taichung, Taiwan. ${ }^{3}$ Department of Chinese Medicine, China Medical University Hospital, Taichung, Taiwan. ${ }^{4}$ Management Office for Health Data, China Medical University Hospital, Taichung, Taiwan. ${ }^{5}$ Department of Respiratory Therapy, China Medical University, Taichung, Taiwan. ${ }^{6}$ Department of Internal Medicine, China Medical University Hospital, Taichung, Taiwan. ${ }^{7}$ Research Center for Traditional Chinese Medicine, Department of Medical Research, China Medical University Hospital, Taichung, Taiwan. ${ }^{8}$ Research Center for Chinese Herbal Medicine, China Medical University, Taichung, Taiwan. ${ }^{9}$ Chinese Medicine Research Center, China Medical University, Taichung, Taiwan. ${ }^{10}$ Department of Biotechnology, Asia University, Taichung, Taiwan.
Received: 31 March 2018 Accepted: 15 November 2018

Published online: 29 November 2018

\section{References}

1. Zumla A, George A, Sharma V, Herbert RH, Oxley A, Oliver M. The WHO 2014 global tuberculosis report--further to go. Lancet Glob Health. 2015; 3(1):e10-2.

2. WHO. WHO Global tuberculosis report. In: Organization WH, editor. In. Switzerland: Geneva; 2014.

3. Centers for Disease Control MoHaW, R.O.C. (Taiwan): CDC Annu Rep 2013-2014. In. Edited by Centers for Disease Control MoHaW. R.O.C (Taiwan): Ministry of Health and Welfare, R.O.C.(Taiwan); 2014.

4. Lee CH, Lee MC, Shu CC, Lim CS, Wang JY, Lee LN, Chao KM. Risk factors for pulmonary tuberculosis in patients with chronic obstructive airway disease in Taiwan: a nationwide cohort study. BMC Infect Dis. 2013;13:194.

5. Chu H, Shih CJ, Lee YJ, Kuo SC, Hsu YT, Ou SM, Shih YN, Tarng DC, Li SY, Chen $Y$ T, et al. Risk of tuberculosis among healthcare workers in an intermediate-burden country: a nationwide population study. J Inf Secur. 2014;69(6):525-32.

6. Cojutti P, Duranti S, Isola M, Baraldo M, Viale P, Bassetti M, Pea F. Might isoniazid plasma exposure be a valuable predictor of drug-related hepatotoxicity risk among adult patients with TB? J Antimicrob Chemother. 2016;71(5):1323-9.

7. Shu CC, Lee CH, Lee MC, Wang JY, Yu CJ, Lee LN. Hepatotoxicity due to first-line anti-tuberculosis drugs: a five-year experience in a Taiwan medical Centre. Int J Tuberc Lung Dis. 2013;17(7):934-9.

8. Saukkonen JJ, Cohn DL, Jasmer RM, Schenker S, Jereb JA, Nolan CM, Peloquin CA, Gordin FM, Nunes D, Strader DB, et al. An official ATS statement: hepatotoxicity of antituberculosis therapy. Am J Respir Crit Care Med. 2006;174(8):935-52.

9. Castro AT, Mendes M, Freitas S, Roxo PC. Incidence and risk factors of major toxicity associated to first-line antituberculosis drugs for latent and active tuberculosis during a period of 10 years. Rev Port Pneumol (2006). 2015; 21(3):144-50.

10. Ramachandran G, Swaminathan S. Safety and tolerability profile of secondline anti-tuberculosis medications. Drug Saf. 2015;38(3):253-69.

11. Yee D, Valiquette C, Pelletier M, Parisien I, Rocher I, Menzies D. Incidence of serious side effects from first-line antituberculosis drugs among patients treated for active tuberculosis. Am J Respir Crit Care Med. 2003;167(11):1472-7.

12. Pasipanodya JG, McNabb SJ, Hilsenrath P, Bae S, Lykens K, Vecino E, Munguia G, Miller TL, Drewyer G, Weis SE. Pulmonary impairment after tuberculosis and its contribution to TB burden. BMC Public Health. 2010;10:259.

13. Shah M, Reed C. Complications of tuberculosis. Curr Opin Infect Dis. 2014; 27(5):403-10.

14. Vecino M, Pasipanodya JG, Slocum P, Bae S, Munguia G, Miller T, Fernandez M, Drewyer G, Weis SE. Evidence for chronic lung impairment in patients treated for pulmonary tuberculosis. J Infect Public Health. 2011:4(5-6):244-52.

15. Falci L, Shi Z, Greenlee H. Multiple chronic conditions and use of complementary and alternative medicine among US adults: results from the 2012 National Health Interview Survey. Prev Chronic Dis. 2016;13:E61.

16. Cheng TY, Chou YJ, Huang N, Pu C, Chou YJ, Chou P. Exploring the role of multiple chronic conditions in traditional Chinese medicine use and three types of traditional Chinese medicine therapy among adults in Taiwan. J Altern Complement Med. 2015;21(6):350-7.

17. Fleischer $T$, Chang $T T$, Chiang $J H$, Hsieh CY, Sun MF, Yen HR. Integration of Chinese herbal medicine therapy improves survival of patients with chronic lymphocytic leukemia: a Nationwide population-based cohort study. Medicine (Baltimore). 2016;95(21):e3788.

18. Moore A, Komesaroff PA, O'Brien K, Xu H, Bensoussan A. Chinese medicine in Australia. J Altern Complement Med. 2016;22(7):515-25.

19. WHO: WHO International Standard Terminologies on Traditional Medicine in the Western Pacific Region In. The LibraryWorld Health Organization Regional Office for the Western PacificP.O. Box 2932 United Nations Ave. cor. Taft Ave. 1000 Manila, Philippines: WHO Regional Office for the Western Pacific; 2007: 356.

20. Chang LC, Huang N, Chou YJ, Lee CH, Kao FY, Huang YT. Utilization patterns of Chinese medicine and Western medicine under the National Health Insurance Program in Taiwan, a population-based study from 1997 to 2003. BMC Health Serv Res. 2008;8:170. 
21. Chen HY, Lin YH, Su H, Chen YC, Yang SH, Chen JL. Investigation on Chinese herbal medicine for primary dysmenorrhea: implication from a nationwide prescription database in Taiwan. Complement Ther Med. 2014; 22(1):116-25.

22. The Regulations of TCM Materials [http://www.mohw.gov.tw/cp-370138847-2.html].

23. Wang M, Guan X, Chi Y, Robinson N, Liu JP. Chinese herbal medicine as adjuvant treatment to chemotherapy for multidrug-resistant tuberculosi (MDR-TB): a systematic review of randomised clinical trials. Tuberculosis (Edinb). 2015;95(4):364-72.

24. Jiang TT, Wang C, Wei LL, Yu XM, Shi LY, Xu DD, Chen ZL, Ping ZP, Li JC. Serum protein gamma-glutamyl hydrolase, Ig gamma- 3 chain $C$ region, and haptoglobin are associated with the syndromes of pulmonary tuberculosis in traditional Chinese medicine. BMC Complement Altern Med. 2015;15:243.

25. Lu J, Ye S, Qin R, Deng Y, Li CP. Effect of Chinese herbal medicine extracts on cell-mediated immunity in a rat model of tuberculosis induced by multiple drug-resistant bacilli. Mol Med Rep. 2013;8(1):227-32.

26. Office of Information Services, Executive Yuan: The Republic of China (Taiwan) - Yearbook 2012. Taiwan, R.O.C.: Executive Yuan, Taiwan; 2012.

27. Welfare MoHa: statistical indicators of Health and Welfare. In. https://dep. mohw.gov.tw/DOS/Ip-2158-113.html: Statistics Department, Ministry of Health and Welfare; 2015

28. Huang TP, Liu PH, Lien AS, Yang SL, Chang HH, Yen HR. A nationwide population-based study of traditional Chinese medicine usage in children in Taiwan. Complement Ther Med. 2014;22(3):500-10.

29. Liu C-Y, Hung Y, Chuang Y, Chen Y, Weng W, Liu J, Liang K. Incorporating development stratification of Taiwan townships into sampling design of large scale health interview survey. J Health Manag. 2006;4(1):1-22.

30. Bensky D, Clavey S, Stoger E: Chinese herbal medicine: Materia Medica 3rd Ed. edn. Seatle, WA, U.S.a.: Eastland press, Inc.; 2004.

31. Scheid V, Bensky D, Ellis A, Barolet R. Chinese herbal medicine: formulas \& strategies. Eastland press; 2009.

32. Chan K, Shaw D, Simmonds MS, Leon CJ, Xu Q, Lu A, Sutherland I, Ignatova S, Zhu YP, Verpoorte R, et al. Good practice in reviewing and publishing studies on herbal medicine, with special emphasis on traditional Chinese medicine and Chinese materia medica. J Ethnopharmacol. 2012;140(3):469-75.

33. Chang CM, Chu HT, Wei YH, Chen FP, Wang S, Wu PC, Yen HR, Chen TJ, Chang $\mathrm{HH}$. The Core pattern analysis on Chinese herbal medicine for Sjogren's syndrome: a Nationwide population-based study. Sci Rep. 2015;5:9541.

34. Shih CC, Liao CC, Su YC, Tsai CC, Lin JG. Gender differences in traditional Chinese medicine use among adults in Taiwan. PLoS One. 2012;7(4):e32540.

35. Tostmann A, Boeree MJ, Aarnoutse RE, de Lange WC, van der Ven AJ, Dekhuijzen R. Antituberculosis drug-induced hepatotoxicity: concise up-todate review. J Gastroenterol Hepatol. 2008;23(2):192-202.

36. Rhines AS. The role of sex differences in the prevalence and transmission of tuberculosis. Tuberculosis (Edinb). 2013;93(1):104-7.

37. Kuo YT, Chang TT, Muo CH, Wu MY, Sun MF, Yeh CC, Yen HR. Use of complementary traditional Chinese medicines by adult Cancer patients in Taiwan: a Nationwide population-based study. Integr Cancer Ther. 2018; 17(2):531-41.

38. Huang MC, Pai FT, Lin CC, Chang CM, Chang HH, Lee YC, Sun MF, Yen HR Characteristics of traditional Chinese medicine use in patients with rheumatoid arthritis in Taiwan: a nationwide population-based study. J Ethnopharmacol. 2015;176:9-16.

39. Lin YR, Wu MY, Chiang JH, Yen HR, Yang ST. The utilization of traditional Chinese medicine in patients with dysfunctional uterine bleeding in Taiwan: a nationwide population-based study. BMC Complement Altern Med. 2017;17(1):427.

40. Yen H, Huang T, Sun M. Chinese medicine usage in Taiwan: a nationwide population-based study. RCHM J. 2013;10:21-7.

41. Lin YJ, Tian WH, Chen CC. Urbanization and the utilization of outpatient services under National Health Insurance in Taiwan. Health Policy. 2011; 103(2-3):236-43.

42. Shih SF, Lew-Ting CY, Chang HY, Kuo KN. Insurance covered and noncovered complementary and alternative medicine utilisation among adults in Taiwan. Soc Sci Med. 2008;67(7):1183-9.

43. Duarte R, Lonnroth K, Carvalho C, Lima F, Carvalho ACC, Munoz-Torrico M, Centis R. Tuberculosis, social determinants and co-morbidities (including HIV). Pulmonology. 2018;24(2):115-9.

44. Tanimura T, Jaramillo E, Weil D, Raviglione M, Lonnroth K. Financial burden for tuberculosis patients in low- and middle-income countries: a systematic review. Eur Respir J. 2014;43(6):1763-75.
45. Murray M, Oxlade O, Lin HH. Modeling social, environmental and biological determinants of tuberculosis. Int J Tuberc Lung Dis. 2011;15(Suppl 2):64-70.

46. Centers for Disease Control MoHaW, R.O.C. (Taiwan): Guidelines for diagnosis and treatment of tuberculosis 5E (in Chinese). In.; 2011: https:// www.cdc.gov.tw/uploads/files/201803/81a3a893-a531-4092-b75558665fe9074a.pdf. Accessed 201824 December 202017.

47. Nathanson E, Gupta R, Huamani P, Leimane V, Pasechnikov AD, Tupasi TE, Vink K, Jaramillo E, Espinal MA. Adverse events in the treatment of multidrug-resistant tuberculosis: results from the DOTS-plus initiative. Int J Tuberc Lung Dis. 2004;8(11):1382-4.

48. Lienhardt C, Cook SV, Burgos M, Yorke-Edwards V, Rigouts L, Anyo G, Kim SJ, Jindani A, Enarson DA, Nunn AJ, et al. Efficacy and safety of a 4-drug fixed-dose combination regimen compared with separate drugs for treatment of pulmonary tuberculosis: the study $\mathrm{C}$ randomized controlled trial. JAMA. 2011;305(14):1415-23.

49. Forget EJ, Menzies D. Adverse reactions to first-line antituberculosis drugs. Expert Opin Drug Saf. 2006:5(2):231-49.

50. Nahin RL, Barnes PM, Stussman BJ, Bloom B. Costs of complementary and alternative medicine (CAM) and frequency of visits to CAM practitioners: United States. Natl Health Stat Report. 2007;2009(18):1-14.

51. Yen HR, Liang KL, Huang TP, Fan JY, Chang TT, Sun MF. Characteristics of traditional Chinese medicine use for children with allergic rhinitis: a nationwide population-based study. Int J Pediatr Otorhinolaryngol. 2015; 79(4):591-7.

52. Yen HR, Sun MF, Lin CL, Sung FC, Wang CC, Liang KL. Adjunctive traditional Chinese medicine therapy for patients with chronic rhinosinusitis: a population-based study. Int Forum Allergy Rhinol. 2015;5(3):240-6.

53. Yu CH, Liu PH, Van YH, Lien AS, Huang TP, Yen HR. Traditional Chinese medicine for idiopathic precocious puberty: a hospital-based retrospective observational study. Complement Ther Med. 2014;22(2):258-65.

54. Wang HM, Lin SK, Yeh CH, Lai JN. Prescription pattern of Chinese herbal products for adult-onset asthma in Taiwan: a population-based study. Ann Allergy Asthma Immunol. 2014;112(5):465-70.

55. Chung KP, Chen JY, Lee CH, Wu HD, Wang JY, Lee LN, Yu CJ, Yang PC, Group T. Trends and predictors of changes in pulmonary function after treatment for pulmonary tuberculosis. Clinics (Sao Paulo). 2011;66(4):549-56.

56. Menezes AM, Hallal PC, Perez-Padilla R, Jardim JR, Muino A, Lopez MV, Valdivia G, Montes de Oca M, Talamo C, Pertuze J, et al. Tuberculosis and airflow obstruction: evidence from the PLATINO study in Latin America. Eur Respir J. 2007;30(6):1180-5.

57. Ryu YJ, Lee JH, Chun EM, Chang JH, Shim SS. Clinical outcomes and prognostic factors in patients with tuberculous destroyed lung. Int J Tuberc Lung Dis. 2011;15(2):246-50 i.

58. Jiang $\mathrm{RH}, \mathrm{Xu} \mathrm{HB}, \mathrm{Fu}$ J. Outcomes of Chinese herb medicine for the treatment of multidrug-resistant tuberculosis: a systematic review and metaanalysis. Complement Ther Med. 2015;23(4):544-54.

59. Lin G, Li P, Li SL, Chan SW. Chromatographic analysis of Fritillaria isosteroidal alkaloids, the active ingredients of Beimu, the antitussive traditional Chinese medicinal herb. J Chromatogr A. 2001;935(1-2):321-38.

60. Wu X, Chan SW, Ma J, Li P, Shaw PC, Lin G. Investigation of association of chemical profiles with the tracheobronchial relaxant activity of Chinese medicinal herb Beimu derived from various Fritillaria species. J Ethnopharmacol. 2017.

61. Xie Y, Sun HX, Li D. Platycodin d improves the immunogenicity of Newcastle disease virus-based recombinant avian influenza vaccine in mice. Chem Biodivers. 2010;7(3):677-89.

62. Kwon OG, Ku SK, An HD, Lee YJ. The effects of Platycodin D, a Saponin purified from Platycodi Radix, on collagen-induced DBA/1J mouse rheumatoid arthritis. Evid Based Complement Alternat Med. 2014;2014:954508.

63. Choi JH, Hwang YP, Lee HS, Jeong HG. Inhibitory effect of Platycodi Radix on ovalbumin-induced airway inflammation in a murine model of asthma. Food Chem Toxicol. 2009:47(6):1272-9.

64. Ryu J, Lee HJ, Park SH, Kim J, Lee D, Lee SK, Kim YS, Hong JH, Seok JH, Lee CJ. Effects of the root of Platycodon grandiflorum on airway mucin hypersecretion in vivo and platycodin $\mathrm{D}(3)$ and deapi-platycodin on production and secretion of airway mucin in vitro. Phytomedicine. 2014;21(4):529-33.

65. Chang HK, Yang HY, Lee TH, Shin MC, Lee MH, Shin MS, Kim CJ, Kim OJ, Hong SP, Cho S. Armeniacae semen extract suppresses lipopolysaccharideinduced expressions of cyclooxygenase [correction of cycloosygenase]-2 and inducible nitric oxide synthase in mouse BV2 microglial cells. Biol Pharm Bull. 2005;28(3):449-54. 
66. Isoza T, Matano Y, Yamamoto K, Kosaka N, Tani T. Quantitative determination of amygdalin epimers by cyclodextrin-modified micellar electrokinetic chromatography. J Chromatogr A. 2001;923(1-2):249-54.

67. Yang HY, Chang HK, Lee JW, Kim YS, Kim H, Lee MH, Shin MS, Ham DH, Park HK, Lee H, et al. Amygdalin suppresses lipopolysaccharide-induced expressions of cyclooxygenase-2 and inducible nitric oxide synthase in mouse BV2 microglial cells. Neurol Res. 2007;29(Suppl 1):S59-64.

68. Lu HM, Liang YZ, Yi LZ, Wu XJ. Anti-inflammatory effect of Houttuynia cordata injection. J Ethnopharmacol. 2006;104(1-2):245-9.

69. Chiow KH, Phoon MC, Putti T, Tan BK, Chow VT. Evaluation of antiviral activities of Houttuynia cordata Thunb. Extract, quercetin, quercetrin and cinanserin on murine coronavirus and dengue virus infection. Asian Pac J Trop Med. 2016;9(1):1-7.

70. Lee HJ, Seo HS, Kim GJ, Jeon CY, Park JH, Jang BH, Park SJ, Shin YC, Ko SG Houttuynia cordata Thunb inhibits the production of pro-inflammatory cytokines through inhibition of the NFkappaB signaling pathway in HMC-1 human mast cells. Mol Med Rep. 2013;8(3):731-6.

71. Minh CV, Nhiem NX, Yen HT, Kiem PV, Tai BH, Le Tuan Anh H, Hien TT, Park S, Kim N, Kim SH. Chemical constituents of Trichosanthes kirilowii and their cytotoxic activities. Arch Pharm Res. 2015;38(8):1443-8.

72. Cai Y, Xiong S, Zheng Y, Luo F, Jiang P, Chu Y. Trichosanthin enhances antitumor immune response in a murine Lewis lung cancer model by boosting the interaction between TSLC1 and CRTAM. Cell Mol Immunol. 2011;8(4): 359-67.

73. Ishibashi H, Mochidome T, Okai J, Ichiki H, Shimada H, Takahama K. Activation of potassium conductance by ophiopogonin-D in acutely dissociated rat paratracheal neurones. Br J Pharmacol. 2001;132(2):461-6.

74. Yao DQ, Tian YP, Gao HB, Gong Y, Meng ZH, Tong F, Shi HW. Study the effects of Salvia miltiorrhiza monomer IH764-3 on the expression of matrix metalloproteinase in lungs of rats exposed to Paraquat (PQ). Zhonghua Lao Dong Wei Sheng Zhi Ye Bing Za Zhi. 2012;30(5):321-5.

75. Lee HJ, Seo M, Lee EJ. Salvianolic acid B inhibits atherogenesis of vascular cells through induction of Nrf2-dependent heme oxygenase-1. Curr Med Chem. 2014;21(26):3095-106.

76. Shang X, He X, Li M, Zhang R, Fan P, Zhang Q, Jia Z. The genus Scutellaria an ethnopharmacological and phytochemical review. J Ethnopharmacol. 2010;128(2):279-313.

77. Zhai HQ, Hhang JR, Gao MC, Liu Y, Zhang SF, Wang XH, Meng FY, Wang YP. Comparative study between Ephedra sinica Stapf and Fructus Schisandrae Chinensis on ET-1 and 6-keto-prostaglandin F1alpha in rats with idiopathic pulmonary fibrosis. Genet Mol Res. 2014;13(2):3761-71.

78. Wan CK, Tse AK, Yu ZL, Zhu GY, Wang H, Fong DW. Inhibition of cytochrome P450 3A4 activity by schisandrol a and gomisin a isolated from Fructus Schisandrae chinensis. Phytomedicine. 2010;17(8-9):702-5.

79. Huang TP, Liu PH, Lien AS, Yang SL, Chang HH, Yen HR. Characteristics of traditional Chinese medicine use in children with asthma: a nationwide population-based study. Allergy. 2013;68(12):1610-3.

80. Kao ST, Yeh TJ, Hsieh CC, Shiau HB, Yeh FT, Lin JG. The effects of ma-Xing-GanShi-tang on respiratory resistance and airway leukocyte infiltration in asthmatic Guinea pigs. Immunopharmacol Immunotoxicol. 2001;23(3):445-58.

81. Lin YC, Chang CW. Wu CR: antitussive, anti-pyretic and toxicological evaluation of ma-Xing-Gan-Shi-tang in rodents. BMC Complement Altern Med. 2016;16(1):456

82. Das AK, Mizuguchi H, Kodama M, Dev S, Umehara H, Kitamura Y, Matsushita C, Takeda N, Fukui H. Sho-seiryu-to suppresses histamine signaling at the transcriptional level in TDl-sensitized nasal allergy model rats. Allergol Int. 2009;58(1):81-8.

83. Nagai T, Arai Y, Emori M, Nunome SY, Yabe T, Takeda T, Yamada H. Antiallergic activity of a Kampo (Japanese herbal) medicine "Sho-seiryu-to (XiaoQing-long-tang)" on airway inflammation in a mouse model. Int Immunopharmacol. 2004:4(10-11):1353-65.

84. Nagai T, Nakao M, Shimizu Y, Kodera Y, Oh-Ishi M, Maeda T, Yamada H: Proteomic analysis of anti-inflammatory effects of a Kampo (Japanese herbal) medicine "Shoseiryuto (Xiao-Qing-long-tang)" on airway inflammation in a mouse model. Evid Based Complement Alternat Med 2011, 2011:604196.

85. Wang SD, Lin LJ, Chen CL, Lee SC, Lin CC, Wang JY, Kao ST. Xiao-Qing-longtang attenuates allergic airway inflammation and remodeling in repetitive Dermatogoides pteronyssinus challenged chronic asthmatic mice model. J Ethnopharmacol. 2012;142(2):531-8.
86. Yang CQ, Sun PY, Ding DZ, Moriuchi H, Ishitsuka Y, Irikura M, Irie T. The ethical Kampo formulation Sho-seiryu-to (TJ-19) prevents bleomycininduced pulmonary fibrosis in rats. Biol Pharm Bull. 2010;33(8):1438-42.

87. Chan CK, Kuo ML, Shen JJ, See LC, Chang HH, Huang JL. Ding Chuan tang, a Chinese herb decoction, could improve airway hyper-responsiveness in stabilized asthmatic children: a randomized, double-blind clinical trial. Pediatr Allergy Immunol. 2006;17(5):316-22.

88. Yang WK, Lee $\mathrm{CH}, \mathrm{Kim}$ MH, Kim SH, Choi HY, Yeo Y, Park YC. Effects of inhalable microparticles of Seonpyejeongcheon-tang in an asthma mouse model: - effects of microparticles of SJT. Aust J Pharm. 2016;19(4):303-11.

89. Minami M, Konishi T, Jiang Z, Arai T, Makino T. Effect of Shin'iseihaito on murine allergic reaction induced by nasal sensitization. J Tradit Complement Med. 2016;6(3):252-6.

90. Wang C, Cao B, Liu QQ, Zou ZQ, Liang ZA, Gu L, Dong JP, Liang LR, Li XW, $\mathrm{Hu} \mathrm{K}$, et al. Oseltamivir compared with the Chinese traditional therapy maxingshigan-yinqiaosan in the treatment of H1N1 influenza: a randomized trial. Ann Intern Med. 2011;155(4):217-25.

91. Tang WR, Yang SH, Yu CT, Wang CC, Huang ST, Huang TH, Chiang MC, Chang YC. Long-term effectiveness of combined treatment with traditional Chinese medicine and Western medicine on the prognosis of patients with lung Cancer. J Altern Complement Med. 2016;22(3):212-22.

\section{Ready to submit your research? Choose BMC and benefit from:}

- fast, convenient online submission

- thorough peer review by experienced researchers in your field

- rapid publication on acceptance

- support for research data, including large and complex data types

- gold Open Access which fosters wider collaboration and increased citations

- maximum visibility for your research: over $100 \mathrm{M}$ website views per year

At $\mathrm{BMC}$, research is always in progress.

Learn more biomedcentral.com/submissions 